\title{
ARTICLE
}

\section{Evaluating the impact of trauma and PTSD on epigenetic prediction of lifespan and neural integrity}

Seyma Katrinli ${ }^{1}$, Jennifer Stevens $\mathbb{i D}^{2}$, Agaz H. Wani ${ }^{3}$, Adriana Lori ${ }^{2}$, Varun Kilaru ${ }^{1}$, Sanne J. H. van Rooij $\mathbb{i D}^{2}$, Rebecca Hinrichs ${ }^{2}$, Abigail Powers ${ }^{2}$, Charles F. Gillespie $\mathbb{D}^{2}$, Vasiliki Michopoulos ${ }^{2}$, Aarti Gautam ${ }^{4}$, Marti Jett $\mathbb{D}^{4}$, Rasha Hammamieh ${ }^{4}$, Ruoting Yang ${ }^{4,5}$ Derek Wildman ${ }^{3}$, Annie $\mathrm{Qu}^{6}$, Karestan Koenen ${ }^{7,8,9}$, Allison E. Aiello ${ }^{10}$, Tanja Jovanovic $\mathbb{D}^{2}$, Monica Uddin ${ }^{3}$, Kerry J. Ressler ${ }^{2,11}$ and $^{2}$ Alicia K. Smith (iD)

Post-traumatic stress disorder (PTSD) is a debilitating disorder that develops in some people following trauma exposure. Trauma and PTSD have been associated with accelerated cellular aging. This study evaluated the effect of trauma and PTSD on accelerated GrimAge, an epigenetic predictor of lifespan, in traumatized civilians. This study included 218 individuals with current PTSD, 427 trauma-exposed controls without any history of PTSD and 209 subjects with lifetime PTSD history who are not categorized as current PTSD cases. The Traumatic Events Inventory (TEI) and Clinician-Administered PTSD Scale (CAPS) were used to measure lifetime trauma burden and PTSD, respectively. DNA from whole blood was interrogated using the MethylationEPIC or HumanMethylation450 BeadChips. GrimAge estimates were calculated using the methylation age calculator. Cortical thickness of 69 female subjects was assessed by using T1-weighted structural MRI images. Associations between trauma exposure, PTSD, cortical thickness, and GrimAge acceleration were tested with multiple regression models. Lifetime trauma burden $(p=0.03)$, current PTSD $(p=0.02)$ and lifetime PTSD $(p=0.005)$ were associated with GrimAge acceleration, indicative of a shorter predicted lifespan. The association with lifetime PTSD was replicated in an independent cohort $(p=0.04)$. In the MRI sub sample, GrimAge acceleration also associated with cortical atrophy in the right lateral orbitofrontal cortex $\left(p_{\text {adj }}=0.03\right)$ and right posterior cingulate $\left(p_{\text {adj }}=0.04\right)$, brain areas associated with emotion-regulation and threat-regulation. Our findings suggest that lifetime trauma and PTSD may contribute to a higher epigenetic-based mortality risk. We also demonstrate a relationship between cortical atrophy in PTSD-relevant brain regions and shorter predicted lifespan.

Neuropsychopharmacology (2020) 45:1609-1616; https://doi.org/10.1038/s41386-020-0700-5

\section{INTRODUCTION}

Traumatic events and posttraumatic stress disorder (PTSD) symptoms may accelerate cellular aging and potentially result in poor health outcomes or even higher mortality risk [1, 2]. Considering high individual and societal costs of PTSD, understanding the impact of trauma and PTSD on lifespan, as well as the neuroanatomical changes associated with this altered lifespan is crucial. Traumatic experiences and PTSD have been suggested to advance cellular aging mainly through oxidative stress and inflammatory pathways [1,3]. Briefly, repeated activation of the hypothalamic-pituitary-adrenal (HPA) axis system by chronic stress and PTSD may cause an elevation in reactive oxygen species and reduce protective capacity of antioxidants, which would increase the risk of neuronal cell death [1]. Moreover, dysregulated glucocorticoid signaling related to PTSD might cause increased inflammation in peripheral and central nervous systems, which could increase risk of necrosis $[3,4]$.
Cellular aging results in highly reliable age-related DNA methylation (DNAm) changes in the epigenome. Studies demonstrate that individuals with DNAm ages that are higher than their chronological age (i.e., accelerated DNAm age) have higher risk of neurocognitive decline [5] and PTSD [6], as well as a shorter lifespan [7].

Recent evidence has raised the possibility that trauma and PTSD may be associated with accelerated DNAm age, which has a negative impact on neural integrity and lifespan $[6,8]$. Multiple cross-sectional studies reported that severity of PTSD and its symptoms associate with advanced DNAm age acceleration $[6,8,9]$. Studies also reported higher DNAm age acceleration with increased trauma burden and number of stressful lifetime events, which are predictors of PTSD [9-12]. A recent longitudinal study conducted on 179 veterans reported a positive association between PTSD symptom scores and DNAm age acceleration [2]. In contrast, Boks et al. reported that PTSD

\footnotetext{
${ }^{1}$ Department of Gynecology and Obstetrics, Emory University, Atlanta, GA, USA; ${ }^{2}$ Department of Psychiatry \& Behavioral Sciences, Emory University, Atlanta, GA, USA; ${ }^{3}$ Genomics Program, College of Public Health, University of South Florida, Tampa, FL, USA; ${ }^{4}$ Integrative Systems Biology, US Army Center for Environmental Health Research, Fort Detrick, MD, USA; ${ }^{5}$ The Geneva Foundation, Fort Detrick, MD, USA; ${ }^{6}$ Department of Statistics, University of Illinois, Champaign, IL, USA; ${ }^{7}$ Stanley Center for Psychiatric Research, Broad Institute of MIT and Harvard, Cambridge, MA, USA; ${ }^{8}$ Psychiatric and Neurodevelopmental Genetics Unit (PNGU), Massachusetts General Hospital, Boston, MA, USA; ${ }^{9}$ Department of

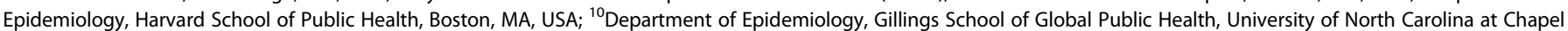
Hill, Chapel Hill, NC, USA and ${ }^{11}$ Department of Psychiatry, McLean Hospital and Harvard Medical School and McLean Hospital, Belmont, MA, USA

Correspondence: Alicia K. Smith (alicia.smith@emory.edu)
}

Received: 23 January 2020 Revised: 17 April 2020 Accepted: 29 April 2020

Published online: 7 May 2020 
symptom severity is associated with reduced DNAm age in 96 Dutch male veterans [11].

Advanced DNAm age also correlates with markers of neural integrity in brain regions known to deteriorate with advanced age [6]. This study, conducted on 281 veterans, reported that DNAmAge acceleration associated with reduced integrity in the genu of the corpus callosum and indirectly related to poorer working memory performance via this region [6]. Over the course of normal aging, a progressive reduction in gray matter volume and cortical thickness, especially in prefrontal regions and cingulate cortex, is imminent [13]. These regions are involved in cognitive functions such as response inhibition and learning [14], which are known to be impaired with advanced age [15]. A recent study demonstrated that the effect of age on cortical thickness was partially mediated by epigenetic signatures [16]. Notably, cortical atrophy is also linked to trauma exposure and PTSD [17].

Recently, Lu et al. developed a novel lifespan predictor, GrimAge, which predicts chronological age in the context of age-related lifestyle factors, such as smoking packs/years [18]. Since GrimAge was trained on time-to-death, it is more predictive of mortality compared to earlier DNAm age predictors that were exclusively trained on chronological age [19]. GrimAge acceleration was validated in 6935 individuals, and increased GrimAge acceleration predicted time-to-death overall and in the context of age-related health problems such as coronary artery disease [18].

No studies to date have examined the association between traumatic stress, cortical atrophy and mortality risk estimators such as GrimAge acceleration. Hence, the primary aim of this study was to evaluate the relationship of trauma and PTSD with mortality risk, using the newly developed GrimAge algorithm. We also sought to examine potential biological links between psychiatric disorder and mortality risk by examining the association between GrimAge acceleration and cortical thickness, a wellknown marker of aging [20,21].

\section{MATERIALS AND METHODS}

\section{Samples}

The individuals included in the study were part of a larger cohort of the Grady Trauma Project (GTP), which investigates the influence of genetic and environmental factors on response to stressful life events in a predominantly African American, urban population of low socioeconomic status [22]. Interviews were conducted with participants in waiting rooms of primary care or obstetrical-gynecological clinics of a large, urban, public hospital in Atlanta, GA. Clinical and life experience information, including PTSD symptoms, trauma exposure, and demographics, along with blood samples have been collected for these subjects. The study was approved by the Institutional Review Board of Emory University School of Medicine and the Grady Health Systems Research Oversight Committee and all participants provided written informed consent.

\footnotetext{
Measures

Demographic information including subject age, sex, and race was provided on a self-administered form. Lifetime exposure to traumatic events was assessed using the Traumatic Events Inventory (TEI) [22, 23]. This self-report instrument measures lifetime (childhood and adulthood) exposure to trauma such as natural disaster, serious accident or injury, and physical or sexual assault, as well as frequency of events, age at worst incident, and feelings of terror, horror, and helplessness. The TEl, which was developed for use with our specific study population, is similar in number of questions and format to other self-report assessments of trauma exposure (see ref. [24, 25] for a review of self-report instruments of civilian trauma exposure). TEI score was operationalized as a continuous measure, ranging from 0 to 16 . For this
}

study, we included only individuals that were exposed to at least one traumatic event (TEl equal or greater than 1).

Childhood trauma exposure was measured using the Childhood Trauma Questionnaire (CTQ), a self-report inventory assessing three types of child abuse: sexual, physical and emotional based on established scores for mild, moderate and severe abuse for each type [26]. History of childhood abuse was operationalized dichotomously, two categories for each type of abuse (physical, sexual, and emotional): (1) those with CTQ scale scores in the none to mild range, and (2) those with CTQ scores in the moderate to severe range. We then created a composite variable across all of the three types of abuse. Using this composite variable, participants were categorized into two groups according to the numbers of types of abuse that fell into the moderate to severe range: (1) those with no type of abuse in the moderate to severe range, and (2) those with at least one type of abuse in the moderate to severe range [27].

PTSD diagnosis was assessed by Clinician-Administered PTSD Scale (CAPS) $[28,29]$. The CAPS provides a diagnostic measure of PTSD and assesses lifetime and current PTSD. For 109 (12.8\%) individuals with missing CAPS information, modified PTSD symptomatic scale (mPSS) based on DSM-IV criteria was used as a measure of current PTSD diagnosis [30]. To be categorized in PTSD group, subjects needed to report current symptoms falling within three symptom clusters: at least 1 intrusive symptom (B), 3 avoidance/numbing symptoms (C), and 2 hyperarousal symptoms (D) on the mPSS, with a duration of 1 month or greater (D) [31]. There was a significant strong positive correlation between mPSS and current CAPS assessment in this study $(r=0.53, p<2.2 \mathrm{e}-16)$, which is consistent with other reports [32]. For both measures used to assess current PTSD, the time between questionnaire completion and blood draw was less than 30 days. Using this PTSD categorization, we identified 218 individuals as current PTSD group (assessed by CAPS or mPSS) (current PTSD group), 427 trauma-exposed individuals without any history of PTSD (control group) and 209 participants with a lifetime PTSD history (assessed by CAPS only), but without current PTSD (assessed by CAPS or mPSS) (lifetime PTSD group). The current PTSD group and lifetime PTSD group were exposed to similar types of traumas, with (1) serious accident or injury and (2) attacked without a weapon by a spouse being the two most common traumas in both groups.

\section{DNA methylation}

DNA was extracted from whole blood and interrogated using the MethylationEPIC BeadChip (Illumina) for 637 samples and HumanMethylation450 BeadChip (Illumina) for 217 samples according to manufacturer's instructions. Raw methylation beta values were determined via GenomeStudio. Samples with probe detection call rates $<90 \%$ and those with an average intensity value of either $<50 \%$ of the experiment-wide sample mean or $<2000$ arbitrary units (AU) were removed using $R$ package CpGassoc [33]. Probes with detection $p$-values $>0.01$ set to missing. CpG sites that cross hybridize between autosomes and sex chromosomes were removed [34].

GrimAge estimates were calculated using the new DNA methylation age calculator developed by Horvath (https://dnamage.genetics. ucla.edu/new) on the non-normalized methylation beta values per the instructions on the website, since the calculator includes a normalization step as a part of its process [18, 35]. For analyses purposes, the age-adjusted version of GrimAge (i.e., AgeAccelGrim variable of the new methylation age calculator output) was used. DNAm patterns were also used to calculate the proportions of CD8T, CD4T, natural killer (NK), B cells, monocytes (mono), and granulocytes. These calculations were done according the methodology described by Houseman et al. [36], using Horvath's new methylation age calculator. Estimated proportions of CD8T, CD4T, NK, B cells, and mono were included as covariates in multiple regression models. 
Image acquisition and processing

To minimize heterogeneity, brain magnetic resonance imaging (MRI) in this sample was restricted to African-American women. $N=69$ study participants from the discovery cohort (GTP) had available and usable MRI data available. Exclusion criteria included no trauma exposure, a history of bipolar disorder, schizophrenia, current psychotic symptoms, current psychotropic medication use, contraindications to MRI scanning, neurological disorder, known structural brain abnormality, head injury with loss of consciousness, implanted metal objects, or positive urine tests for pregnancy and drug use (cocaine, marijuana, opiates, amphetamines, and methamphetamines) assessed 24-48 h before the MRI scan.

Brain imaging data were acquired on a Siemens 3.0-Tesla Magnetom Trio TIM whole-body MR scanner (Siemens, Malvern, PA) with a 12-channel head coil. Structural images were acquired using the Siemens 3D-MP-RAGE T1-weighted pulse sequence (176 slices, TR $=2000 \mathrm{~ms}$, TE $=3.02 \mathrm{~ms} ; 1 \mathrm{~mm}^{3}$ voxel size). The images were processed in Freesurfer version 5.3 (https://surfer. nmr.mgh.harvard.edu).

Cortical surface reconstruction and thickness measurement were performed using Freesurfer's surface-based analysis, and data quality checks were performed following the ENIGMA 3 protocol. Here, summary statistics and a summary of outliers \pm 3 $S D$ from the mean were generated from the thickness values of 70 regions defined by the Desikan Atlas [37]. The raters (SVR and JSS) visually examined each subject's data from both internal (axial, coronal, and sagittal slices) and external views (3D rendering of surface) to identify surface reconstruction errors. Regions that were confirmed to contain an error were discarded. Regional thickness values were exported for further analysis.

\section{Statistical analyses}

We first tested the correlation between chronological age and GrimAge. Age-adjusted version of GrimAge, named as GrimAge acceleration, was calculated by regressing chronological age out from GrimAge. Since GrimAge is a predictor of mortality [18], higher GrimAge acceleration values reflect increased mortality risk.

We then conducted univariate tests to evaluate the association between GrimAge acceleration and potential confounding variables (array type and estimated cell proportions). To evaluate the association of lifetime trauma exposure, childhood trauma, and PTSD (current and lifetime) with GrimAge acceleration, we performed series of multiple regressions, adjusting for sex, array type, and estimated cell proportions. Then, we performed a Kruskal-Wallis test to compare difference in mean GrimAge acceleration among groups, followed by post hoc pairwise comparisons using a Wilcoxon test. Power analysis were conducted using G*Power 3.1 [38].

We examined the association between cortical thickness and GrimAge acceleration in female participants with available MRI data. DNAm was assessed with MethylationEpic BeadChip for all 69 individuals. We used a multiple linear regression model to evaluate GrimAge acceleration as a predictor of cortical thickness, controlling for age. To investigate whether association between GrimAge acceleration and cortical thickness is similar across current PTSD group and controls, we performed stratified analysis for PTSD status. $p$-values were adjusted for multiple-testing of 70 regions by controlling the false discovery rate (FDR) at 5\% [39]. For all statistical analyses beta estimates (effect sizes) are reported alongside with $p$-values.

\section{Replication analysis}

To test replicability of our results, we used data from the Detroit Neighborhood Health Study (DNHS), comprised of predominantly African American adults living in Detroit, Michigan [40, 41]. DNA methylation data was available from 309 unique DNHS participants, 120 of whom met criteria for lifetime PTSD and 189 of whom were trauma-exposed controls without any history of PTSD.
The PTSD Checklist Civilian Version (PCL-C) was used to assess PTSD, as previously described [40]. Number of traumatic event types, assessed using a survey of 19 traumatic events, which can be divided into four groups based on type: assaultive violence, other injury or shocking experience, learning about trauma to a loved one, and sudden unexpected death of a loved one, was used to measure trauma burden [40-42]. MethylationEPIC BeadChip (Illumina) and HumanMethylation450 BeadChip (Illumina) were used to measure whole blood-based DNA methylation, following the manufacturer's recommended protocol. Raw DNA methylation beta values were obtained using GenomeStudio and subsequent quality control processing steps and statistical analysis were performed as described for the GTP samples.

\section{RESULTS}

Demographics of the cohorts

A total of 854 subjects from GTP were included in the study, including 427 trauma-exposed controls without any history of PTSD (control group), 218 individuals with current PTSD, and 209 trauma-exposed individuals who do not meet current PTSD criteria but had a previous history of PTSD (lifetime PTSD group). Demographics and characteristics of the cohort are shown in Table 1. The cohort was predominantly African American (93.1\%) and female (70.0\%)

Demographic and clinical characteristics of the replication cohort from DNHS, including 120 individuals with lifetime PTSD and 189 trauma-exposed controls without any history of PTSD, are shown in Supplementary Table 1.

Associations between GrimAge acceleration and demographic and cellular variables

GrimAge was correlated with chronological age $(r=0.82, p<2.2 \mathrm{e}$ $-16)$, but GrimAge acceleration was not correlated with chronological age $(r=-0.002, p=0.96)$. We then compared GrimAge with other estimates of DNAmAge. Even though GrimAge was correlated with Horvath's $(r=0.74, p<2.2 \mathrm{e}-16)$ and Hannum's $(r=0.85, p<2.2 \mathrm{e}-16)$ DNAmAge, the degree of correlation between the residuals of the epigenetic clocks (GrimAge acceleration and other DNAmAge acceleration estimates) were lower for both the Horvath $(r=0.07, p=0.04)$ and Hannum $(r=0.18, p=$ $1.66 \mathrm{e}-9)$ estimates.

Supplementary Table 2 shows the results of univariate associations between GrimAge acceleration and sex, array type, and blood cell proportions in discovery and replication cohorts. There were significant associations between blood cell proportions and GrimAge acceleration. CD8T, CD4T, NK, and B cell proportions were negatively associated with GrimAge acceleration, whilst monocytes and granulocytes were positively associated. Similar effects for cell proportions in GrimAge were also observed in the replication DNHS cohort (Supplementary Table 2). Array type was associated with GrimAge acceleration in DNHS cohort and was included as a covariate.

\section{Association between trauma variables, PTSD, and GrimAge}

The results for the regression of GrimAge acceleration on trauma variables (lifetime and childhood trauma) and PTSD in discovery and replication cohorts are summarized on Table 2 . There was no significant difference in GrimAge estimates between current PTSD and control groups $(t=1.30, p=0.20)$. However, GrimAge acceleration differed significantly between current PTSD and control groups $(t=2.25, p=0.02)$. We also noted an association between any PTSD (current or lifetime) and GrimAge acceleration $(t=2.80, p=0.005)$. This association was replicated in an independent cohort $(t=2.04, p=0.04)$, with lifetime PTSD group having advanced GrimAge acceleration relative to controls without any history of PTSD, suggesting an increased mortality risk for individuals with PTSD. In addition, participants who experienced 
Evaluating the impact of trauma and PTSD on epigenetic prediction of...

Table 1. Demographic characteristics of the study $(N=854)$.

\begin{tabular}{lcccc}
\hline Phenotype & Mean $(S D)$ or $N(\%)$ & & \\
\cline { 2 - 4 } & Control $(N=427)$ & Current PTSD $(N=218)$ & Lifetime PTSD (N=209) \\
\hline Sex, Female & $283(66.1 \%)$ & $174(79.8 \%)$ & $138(66.0 \%)$ & 0.0008 \\
Age & $42.80(12.70)$ & $40.93(11.43)$ & $43.30(11.80)$ & $190(90.9 \%)$ \\
Race, AA & $404(94.6 \%)$ & $201(92.2 \%)$ & $47.94(12.33)$ & 0.07 \\
GrimAge & $46.17(12.79)$ & $44.89(11.32)$ & $0.41(4.91)$ & 0.20 \\
GrimAge acceleration & $-0.37(4.85)$ & $0.47(4.82)$ & $5.92(3.08)$ & $107(51.2 \%)$
\end{tabular}

The $p$-values were computed from Kruskal-Wallis test (for continuous variables) and chi-square test (for categorical variables) for comparison of control, current PTSD, and lifetime PTSD groups.

$S D$ standard deviation, $A A$ African American.

a Measured by Traumatic Events Inventory (TEI).

${ }^{\mathrm{b}}$ Measured by Childhood Trauma Inventory (CTQ).

Table 2. Association between trauma variables, PTSD and GrimAge acceleration.

\begin{tabular}{|c|c|c|c|c|c|c|c|c|}
\hline Childhood trauma & 0.19 & 0.37 & 0.51 & 0.61 & -0.65 & 0.85 & -0.76 & 0.44 \\
\hline Current PTSD ${ }^{a}$ & 0.86 & 0.38 & 2.25 & 0.02 & NA & NA & NA & NA \\
\hline Lifetime PTSD ${ }^{b}$ & 0.87 & 0.31 & 2.80 & 0.005 & 1.38 & 0.67 & 2.04 & 0.04 \\
\hline
\end{tabular}

SE standard error

Results are from multiple regression, controlling for sex, array type and cell proportions of CD4T, CD8T, NK, B cell, mono. Significant effects are shown in bold. a Data not available for DNHS cohort.

${ }^{\mathrm{b}}$ Assessed by CAPS in GTP, and by PCL-C in DNHS.

increased lifetime trauma burden had accelerated GrimAge in the GTP discovery cohort (beta estimate $=0.12, p=0.03$ ), indicating higher risk of mortality (Fig. 1a). Even though the association between lifetime trauma burden and GrimAge acceleration had a similar trend in the replication cohort (Table 2), it was not statistically significant (beta estimate $=0.13, p=0.06$ ). There was no association between childhood trauma and GrimAge acceleration in both cohorts. This likely reflects a lack of power, as our post-hoc analysis showed substantially lower power for childhood (8\%) trauma relative to either PTSD (62\%) or lifetime trauma (57\%).

To evaluate whether the PTSD-related increase in GrimAge acceleration may be reversible, we compared the difference in mean GrimAge acceleration among groups using Kruskal-Wallis test and performed post hoc pairwise comparisons using a Wilcoxon test (Fig. 1b). The control group without any history of PTSD had significantly lower GrimAge acceleration compared to both the current PTSD group $(p=0.015)$ and the lifetime PTSD group $(p=0.026)$. GrimAge acceleration did not differ between the current PTSD and lifetime PTSD group ( $p=0.95)$, suggesting that the effect of PTSD on accelerated GrimAge may be permanent, at least when assessed in this manner.

Accelerated GrimAge and cortical thickness

The demographics of the 69 individuals from the GTP cohort (26 individuals from the current PTSD group and 43 individuals from the control group) with available MRI data and cortical thickness measures of 70 regions were shown in Supplementary Table 3. We observed reduced thickness in eight cortical regions with accelerated GrimAge (Table 3). The results of all 70 regions were shown in Supplementary Table 4. Among these tests, two cortical regions are still significant after correction for multiple testing
(FDR < 0.05): GrimAge acceleration was negatively associated with thickness of right lateral orbitofrontal cortex (beta estimate $=$ $-0.014, p_{\text {adj }}=0.03$ ) and right posterior cingulate cortex (beta estimate $=-0.012, p_{\text {adj }}=0.04$ ) (Table 3, Fig. 2). We then tested the association between GrimAge acceleration and cortical thickness in current PTSD group and controls separately. Interestingly, accelerated GrimAge was negatively associated with thickness of the right lateral orbitofrontal cortex in the current PTSD group (beta estimate $=-0.024, p_{\text {adj }}=0.05$ ), but not in the control group.

\section{DISCUSSION}

Traumatic experiences and PTSD have high individual and societal costs. Therefore, understanding the effects of trauma and traumarelated outcomes (e.g., PTSD) on individuals' lifespan and mortality risk is crucial. Here, we evaluated the associations between trauma exposure (both lifetime and childhood), PTSD, and mortality, using the newly developed epigenetic clock GrimAge, which is a DNAmbased biomarker of mortality [18]. One of the strengths of GrimAge is that it incorporates methylation-based proxies of smoking packs per year and metabolism-related plasma proteins, which may be more reliable than adjusting for the self-reported information. Results of this study indicated that lifetime but not childhood trauma exposure and PTSD may associate with increased GrimAge acceleration, implicating shorter life span, and accelerated GrimAge was also related to cortical atrophy.

Findings of this study are consistent with the hypothesis that PTSD is associated with advanced cellular aging and DNAm age, and that advanced DNAm age is related to reduced neural integrity and cognitive ability [6]. Our findings are also consistent with previous studies that used telomere length as a marker of 


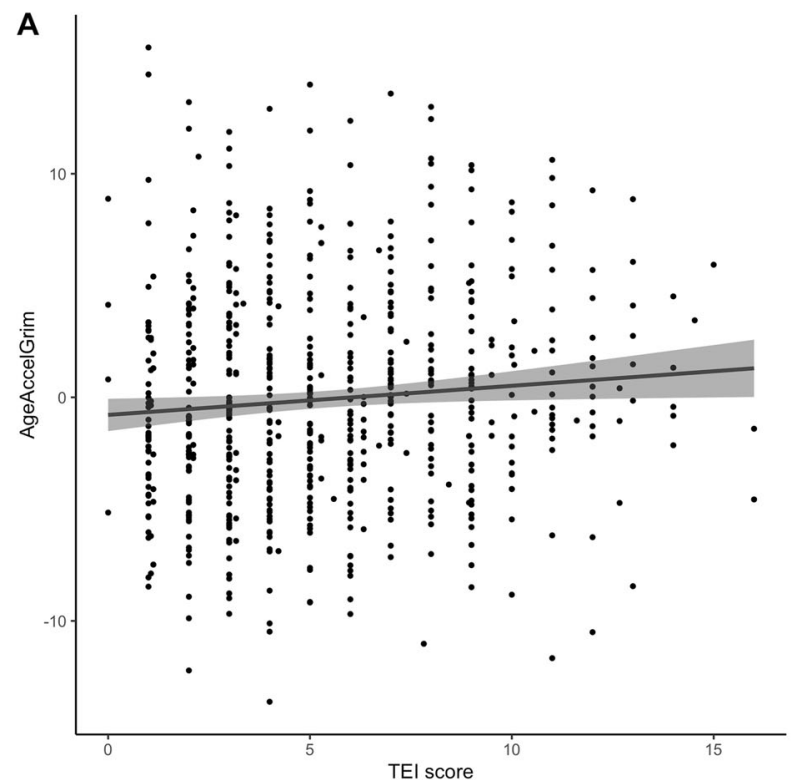

B

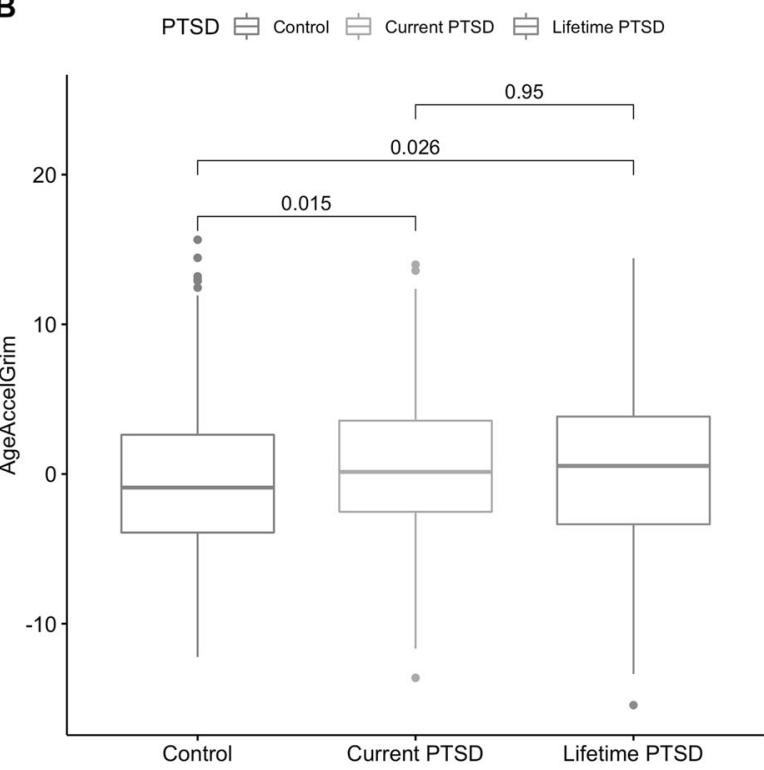

Fig. 1 Associations between GrimAge acceleration and (a) Lifetime trauma and (b) PTSD. a Lifetime trauma is assessed by Traumatic Events Inventory (TEI) score. Red line shows linear best fit for the multiple regression model that includes CD8T, CD4T, NK, B cell, mono, sex and array type as covariates. Blue shaded area represents $95 \%$ confidence region. b Results are $p$ values from Wilcoxon test for post hoc pairwise comparisons.

\begin{tabular}{|c|c|c|c|c|c|c|c|c|c|c|c|c|}
\hline Cortical thickness & Beta & SE & $p$ value & $p_{\text {adj }}$ & Beta & SE & $p$ value & $p_{\text {adj }}$ & Beta & SE & $p$ value & $p_{\text {adj }}$ \\
\hline R lateral OFC & -0.014 & 0.004 & $4.29 \mathrm{E}-04$ & 0.03 & -0.024 & 0.006 & 0.001 & 0.05 & -0.010 & 0.005 & 0.058 & 1 \\
\hline $\mathrm{R}$ posterior $\mathrm{CC}$ & -0.012 & 0.004 & 0.001 & 0.04 & -0.011 & 0.007 & 0.128 & 0.37 & -0.013 & 0.004 & 0.003 & 0.2 \\
\hline $\mathrm{R}$ caudal anterior $\mathrm{CC}$ & -0.018 & 0.007 & 0.013 & 0.25 & -0.004 & 0.014 & 0.754 & 0.87 & -0.025 & 0.010 & 0.020 & 0.7 \\
\hline $\mathrm{R}$ pars orbitalis & -0.014 & 0.006 & 0.021 & 0.25 & -0.009 & 0.008 & 0.261 & 0.47 & -0.011 & 0.005 & 0.032 & 0.8 \\
\hline L caudal anterior CC & -0.019 & 0.008 & 0.022 & 0.25 & -0.025 & 0.011 & 0.037 & 0.24 & -0.009 & 0.007 & 0.205 & 1 \\
\hline L lateral OFC & -0.009 & 0.004 & 0.028 & 0.28 & -0.012 & 0.007 & 0.079 & 0.29 & -0.007 & 0.005 & 0.188 & 1 \\
\hline Right caudal MFC & -0.009 & 0.004 & 0.048 & 0.37 & -0.016 & 0.009 & 0.090 & 0.30 & -0.004 & 0.005 & 0.350 & 1 \\
\hline
\end{tabular}

aging, reporting association of PTSD with shortening of telomere length $[3,43]$, which is also related to reduced brain volume [44]. We also showed that GrimAge acceleration is higher in subjects with a lifetime history of PTSD, compared to controls. There was no significant difference between GrimAge acceleration of subjects with a lifetime PTSD history and subjects with current PTSD, suggesting that PTSD-associated epigenetic marks may be persistent and may still continue to impact lifespan.

Our neuroimaging analyes were focused on cortical thickness, a well-known marker of aging [13]. We showed that accelerated GrimAge was associated with thinner right orbitofrontal cortex (OFC) and right posterior cingulate cortex (PCC), which get thinner with advanced chronological age. These effects were observed above and beyond effects of chronological age, which was included as a covariate in the model, and thus represent a brain index of greater-than-expected progression towards mortality. Interestingly, atrophy in these regions has been reported to be associated with both PTSD and trauma [17]. Thinner right OFC has also previously been associated with metabolic syndrome in combat veterans with PTSD, an effect postulated to reflect cooccurring neurodegenerative and cardiometabolic effects of accelerated aging [45]. The current findings also support this possibility, especially considering that the negative association between GrimAge acceleration and OFC thickness was observed in the current PTSD group $(N=26)$, but not in the control group $(N=43)$.

Background age-related decreases in cortical thickness are typically quite small, at a rate of $0.1-0.5 \%$ per year in adults across ages 20-90 [46]. The effect sizes for GrimAge effects on thickness in the right OFC and PCC reflects 0.014 and $0.012 \mathrm{~mm}$ decrease, respectively, for each year of GrimAge acceleration; indicating $0.6 \%(0.014 \mathrm{~mm}$ over $2.19 \mathrm{~mm}$ mean thickness) and $0.5 \%(0.012$ $\mathrm{mm}$ over $2.37 \mathrm{~mm}$ mean thickness) change in thickness relative to expected age-related change-a marked increase beyond standard age-related effects. A longitudinal study with a similar mean age of our cohort $\left(M_{\mathrm{age}}=50\right.$ years) reported change in thickness 

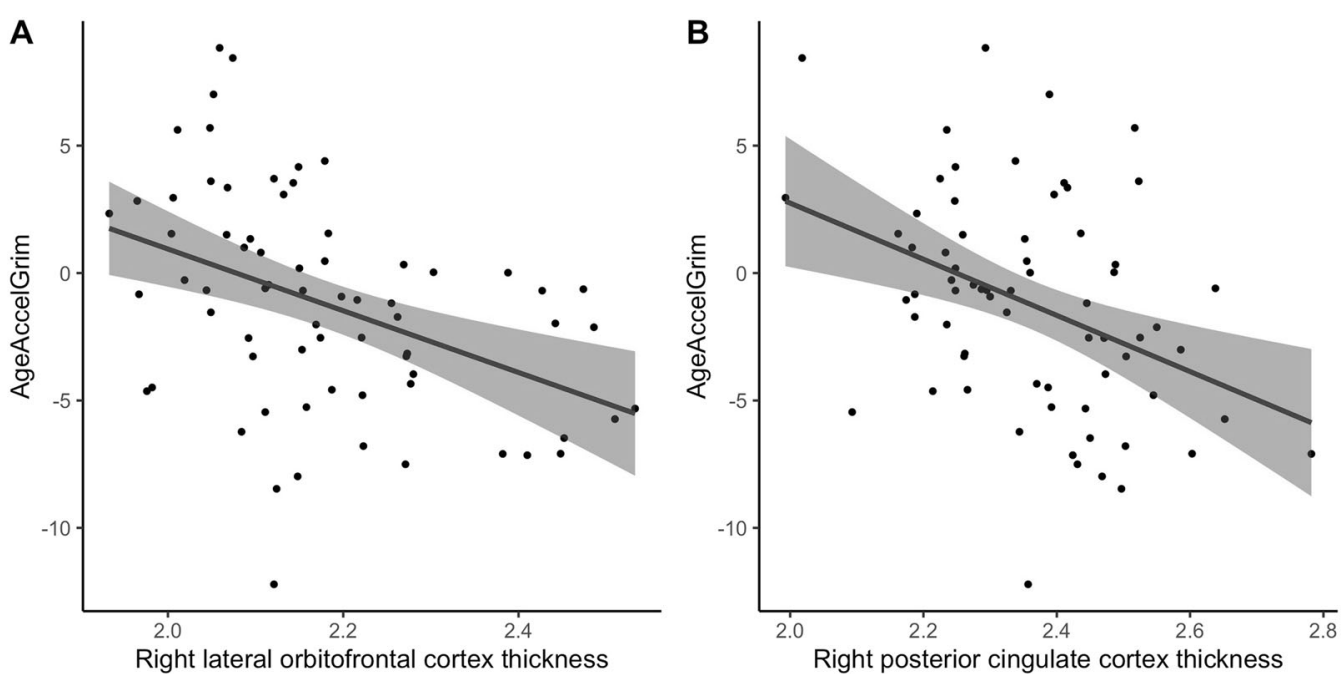

Fig. 2 Associations between GrimAge acceleration and (a) Right lateral orbitofrontal cortex and (b) Right posterior cingulate cortex thickness. a Red line shows linear best fit for the multiple regression model that includes right lateral orbitofrontal thickness as dependent variable, GrimAge as predictor and age as a covariate. Blue shaded area represents $95 \%$ confidence region. b Red line shows linear best fit for the multiple regression model that includes right posterior cingulate thickness as dependent variable, GrimAge as predictor and age as a covariate. Blue shaded area represents $95 \%$ confidence region.

for the OFC $(-0.32 \%$ to $-0.48 \%)$ and the PCC $(-0.41 \%)$ per year [47]. Our findings suggest that these rates almost the double over and above expected age-related decline. The functions of these regions are relevant to PTSD as well as mood disorders and agerelated neurodegenerative conditions. The OFC plays a key role in reward and aversive valuation, participating in a circuit with the ventral striatum that supports stimulus-reinforcement associative learning [48]. Lower OFC volume has consistently been linked with major depressive disorder [49], and geriatric depression [50]. The PCC, in contrast, is a multi-modal association area that receives visual sensory input and is involved in spatial orientation and memory, as well as social cognition [14], and is a hub for the default mode network with high metabolic demand [51]. Lower PCC thickness is a notable feature of neurodegenerative conditions including advanced mild cognitive impairment (MCl) [52], and both typical and atypical presentations of Alzheimer's Disease [53]. The effect of GrimAge acceleration on thickness in these regions is therefore largely consistent with effects of neurodegenerative disorders that are common in older populations.

It is also important to note that our analysis of cell type differences and GrimAge acceleration showed a positive correlation with granulocytes and monocytes. Neutrophils, the most abundant type of granulocytes, and monocytes play important roles in inflammation (reviewed in $[54,55])$. To date, multiple studies report that indicators of inflammation, such as the neutrophil-tolymphocyte ratio or higher granulocyte and monocyte counts, associate with all-cause and cause-specific mortality [56-59]. Current evidence also supports the involvement of inflammation in PTSD [60]. Thus, inflammation may be a key factor in the relationship between PTSD and GrimAge acceleration.

The results of the current study should be considered with a number of limitations. First, this was a cross-sectional study and we are not able to make causal interpretations, but the association between PTSD and GrimAge was replicated in an independent cohort. We observed a positive trend in same direction for the relationship between lifetime trauma burden and accelerated GrimAge. However, it did not reach a statistical significance, potentially because of the difference in lifetime trauma assessments and burden in the respective cohorts (i.e., DNHS did not assess frequency of exposure to various trauma types, as was performed in the GTP). Given the cross-sectional nature of the study, we do not have follow-up data on participants, including information regarding development of age-related disorders, time of death, or future PTSD recurrence in the lifetime PTSD group. Hence, we acknowledge the design of this group is not ideal. Future prospective studies can address this limitation and test whether the effect of PTSD on accelerated GrimAge may be permanent. We were also not able to test the prediction accuracy of GrimAge, or previous epigenetic clock estimates, in terms of mortality risk as that data is not available in our cohort. Future prospective studies are required to evaluate risk of mortality, and its association with GrimAge acceleration and PTSD, as well as to test the hypothesis that the effect of PTSD on accelerated GrimAge may be permanent. Second, the cohort comprised predominantly African American women. Considering recent evidence suggesting that epigenetic clocks including GrimAge may differ by sex $[7,8,18]$ and ethnicity [61], further research in more heterogenous cohort is needed. Third, our sample size for the neuroimaging analysis is relatively small $(N=69)$; thus, we are underpowered to conduct moderation tests of PTSD groups, cortical thickness and GrimAge acceleration, which would be useful to further characterize the observed associations.

Elucidating the molecular mechanisms that link trauma exposure and PTSD to negative biological outcomes is crucial. Even though prior evidence reported substantial comorbidities [62] and accelerated DNAm age associated with PTSD [6, 8, 9], we found only a few reports regarding the relationship between PTSD and mortality risk [63], mostly due the requirement of prospective studies with long-term follow-up. However, the recently developed epigenetic clock GrimAge allows the interpretation of mortality risk using cross sectional-data [18].

Findings from this study shows the association between lifetime trauma burden, PTSD, and accelerated GrimAge and indicate that lifetime trauma exposure and PTSD negatively impact lifespan. Our results also link PTSD relevant cerebral cortex regions, lateral orbitofrontal cortex and posterior cingulate, to accelerated GrimAge and suggest a plausible relationship between reduced cerebral cortex thickness and mortality risk. Further studies are required to support the validity of GrimAge. After gathering supporting results regarding the accuracy of GrimAge and validating the associations between trauma, PTSD and accelerated GrimAge in independent cohorts, this novel measure might be clinically useful to inform who is at greatest risk for increased mortality in the aftermath of trauma. 


\section{FUNDING AND DISCLOSURE}

This research was funded by grants from the National Institute of Mental Health MH096764 (KJR), MH071537 (CFG), RC1MH088283 (AEA); from the National Institute on Drug Abuse R01DA022720 (AEA), R01DA022720-S1 (AEA); and from the National Institute on Minority Health and Health Disparities R01MD011728 (AEA, MU, and DW) and R01MD011728-S2 (AEA, MU, and DW). The content is solely the responsibility of the authors and does not necessarily represent the official views of the National Institutes of Health.

The views, opinions, and/or findings contained in this report are those of the authors and should not be construed as official Department of the Army position, policy, or decision, unless so designated by other official documentation. Citations of commercial organizations or trade names in this report do not constitute an official Department of the Army endorsement or approval of the products or services of these organizations. All authors report no financial interests or potential conflicts of interest. The authors declare no competing interests.

\section{ACKNOWLEDGEMENTS}

We appreciate the technical support of all of the staff, volunteers and participants from the Grady Trauma Project and the Detroit Neighborhood Health Study.

\section{AUTHOR CONTRIBUTIONS}

AKS conceived and executed the parent project with $\mathrm{KJR}$, and participated fully in the writing of this manuscript. SK conducted all analysis and wrote the initial draft with AKS and JS. RH, AP, CFG, VM, TJ, and KJR collected the data, provided clinical expertize and served as study physicians for GTP cohort. KK and AEA collected the data, provided clinical expertize and served as study physicians for DNHS cohort. JS and SJHR performed the neuroimaging analysis. $A G, M J, R H$, and RY conducted methylation analysis of GTP and contributed in the quality control of the biological assays. AL and VK participated in quality control analysis of GTP methylation dataset. AHW performed replication analysis in DNHS cohort with DW, $A Q$, and MU. All authors reviewed and edited the final manuscript.

\section{ADDITIONAL INFORMATION}

Supplementary Information accompanies this paper at (https://doi.org/10.1038/ s41386-020-0700-5)

Publisher's note Springer Nature remains neutral with regard to jurisdictional claims in published maps and institutional affiliations.

\section{REFERENCES}

1. Miller MW, Sadeh N. Traumatic stress, oxidative stress and post-traumatic stress disorder: neurodegeneration and the accelerated-aging hypothesis. Mol Psychiatry. 2014;19:1156-62.

2. Wolf EJ, Logue MW, Morrison FG, Wilcox ES, Stone A, Schichman SA, et al. Posttraumatic psychopathology and the pace of the epigenetic clock: a longitudinal investigation. Psychol Med. 2019;49:791-800.

3. Lohr JB, Palmer BW, Eidt CA, Aailaboyina S, Mausbach BT, Wolkowitz OM, et al. Is post-traumatic stress disorder associated with premature senescence? A review of the literature. Am J Geriatr Psychiatry. 2015;23:709-25.

4. Williamson JB, Porges EC, Lamb DG, Porges SW. Maladaptive autonomic regulation in PTSD accelerates physiological aging. Front Psychol. 2014;5:1571.

5. Levine ME, Lu AT, Bennett DA, Horvath S. Epigenetic age of the pre-frontal cortex is associated with neuritic plaques, amyloid load, and Alzheimer's disease related cognitive functioning. Aging. 2015;7:1198-211.

6. Wolf EJ, Logue MW, Hayes JP, Sadeh N, Schichman SA, Stone A, et al. Accelerated DNA methylation age: associations with PTSD and neural integrity. Psychoneuroendocrinology. 2016;63:155-62.

7. Marioni RE, Shah S, McRae AF, Chen BH, Colicino E, Harris SE, et al. DNA methylation age of blood predicts all-cause mortality in later life. Genome Biol. 2015;16:25.

8. Wolf EJ, Logue MW, Stoop TB, Schichman SA, Stone A, Sadeh N, et al. Accelerated DNA methylation age: associations with PTSD and mortality. Psychosom Med. 2017;80:42-48.
9. Wolf EJ, Maniates $H$, Nugent $N$, Maihofer AX, Armstrong D, Ratanatharathorn A, et al. Traumatic stress and accelerated DNA methylation age: a meta-analysis. Psychoneuroendocrinology. 2018;92:123-34.

10. Zannas AS, Arloth J, Carrillo-Roa T, lurato S, Roh S, Ressler KJ, et al. Lifetime stress accelerates epigenetic aging in an urban, African American cohort: relevance of glucocorticoid signaling. Genome Biol. 2015;16:266.

11. Boks MP, van Mierlo HC, Rutten BP, Radstake TR, De Witte L, Geuze E, et al. Longitudinal changes of telomere length and epigenetic age related to traumatic stress and post-traumatic stress disorder. Psychoneuroendocrinology. 2015;51: 506-12.

12. Sumner JA, Colich NL, Uddin M, Armstrong D, McLaughlin KA. Early experiences of threat, but not deprivation, are associated with accelerated biological aging in children and adolescents. Biol Psychiatry. 2019;85:268-78.

13. Salat DH, Buckner RL, Snyder AZ, Greve DN, Desikan RS, Busa E, et al. Thinning of the cerebral cortex in aging. Cereb Cortex. 2004;14:721-30.

14. Maddock RJ, Garrett AS, Buonocore MH. Remembering familiar people: the posterior cingulate cortex and autobiographical memory retrieval. Neuroscience. 2001;104:667-76.

15. Park DC, Reuter-Lorenz P. The adaptive brain: aging and neurocognitive scaffolding. Annu Rev Psychol. 2009;60:173-96.

16. Freytag V, Carrillo-Roa T, Milnik A, Samann PG, Vukojevic V, Coynel D, et al. A peripheral epigenetic signature of immune system genes is linked to neocortical thickness and memory. Nat Commun. 2017;8:15193.

17. Lindemer ER, Salat DH, Leritz EC, McGlinchey RE, Milberg WP. Reduced cortical thickness with increased lifetime burden of PTSD in OEF/OIF Veterans and the impact of comorbid TBI. Neuroimage Clin. 2013;2:601-11.

18. Lu AT, Quach A, Wilson JG, Reiner AP, Aviv A, Raj K, et al. DNA methylation GrimAge strongly predicts lifespan and healthspan. Aging. 2019;11:303-27.

19. Zhang Q, Vallerga CL, Walker RM, Lin T, Henders AK, Montgomery GW, et al. Improved precision of epigenetic clock estimates across tissues and its implication for biological ageing. Genome Med. 2019;11:54.

20. Resnick SM, Pham DL, Kraut MA, Zonderman AB, Davatzikos C. Longitudinal magnetic resonance imaging studies of older adults: a shrinking brain. J Neurosci. 2003;23:3295-301.

21. Good CD, Johnsrude IS, Ashburner J, Henson RN, Friston KJ, Frackowiak RS. A voxel-based morphometric study of ageing in 465 normal adult human brains. Neuroimage. 2001;14:21-36.

22. Gillespie CF, Bradley B, Mercer K, Smith AK, Conneely K, Gapen M, et al. Trauma exposure and stress-related disorders in inner city primary care patients. Gen Hosp Psychiatry. 2009;31:505-14.

23. Binder EB, Bradley RG, Liu W, Epstein MP, Deveau TC, Mercer KB, et al. Association of FKBP5 polymorphisms and childhood abuse with risk of posttraumatic stress disorder symptoms in adults. JAMA. 2008;299:1291-305.

24. Cusack KJ, Frueh BC, Brady KT. Trauma history screening in a community mental health center. Psychiatr Serv. 2004;55:157-62.

25. Norris F, Hamblen J. Standardized self-report measures of civilian trauma and PTSD. In: Wilson JP, Terence KM, editors. Assessing psychological trauma and PTSD. New York: The Guilford Press; 2004.

26. Bernstein DP, Fink L. Childhood trauma questionnaire: a retrospective self-report manual. San Antonio, TX: The Psychological Corporation; 1998.

27. Bradley RG, Binder EB, Epstein MP, Tang Y, Nair HP, Liu W, et al. Influence of child abuse on adult depression: moderation by the corticotropin-releasing hormone receptor gene. Arch Gen Psychiatry. 2008;65:190-200.

28. Blake DD, Weathers FW, Nagy LM, Kaloupek DG, Gusman FD, Charney DS, et al. The development of a Clinician-administered PTSD scale. J Trauma Stress. 1995;8:75-90.

29. Weathers FW, Keane TM, Davidson JR. Clinician-administered PTSD scale: a review of the first ten years of research. Depress Anxiety. 2001;13:132-56.

30. Falsetti SA, Resnick HS, Resick PA, Kilpatrick DG. The modified PTSD symptom scale: a brief self-report measure of posttraumatic stress disorder. Behav Therapist. 1993;16:161-62.

31. Jovanovic T, Norrholm SD, Blanding NQ, Davis M, Duncan E, Bradley B, et al. Impaired fear inhibition is a biomarker of PTSD but not depression. Depress Anxiety. 2010;27:244-51.

32. Foa EB, Tolin DF. Comparison of the PTSD Symptom Scale-Interview Version and the Clinician-Administered PTSD scale. J Trauma Stress. 2000;13:181-91.

33. Barfield RT, Kilaru V, Smith AK, Conneely KN. CpGassoc: an R function for analysis of DNA methylation microarray data. Bioinformatics. 2012;28:1280-1.

34. McCartney DL, Walker RM, Morris SW, Mclntosh AM, Porteous DJ, Evans KL. Identification of polymorphic and off-target probe binding sites on the Illumina Infinium MethylationEPIC BeadChip. Genom Data. 2016;9:22-4.

35. Horvath S. DNA methylation age of human tissues and cell types. Genome Biol. 2013;14:R115. 
36. Houseman EA, Accomando WP, Koestler DC, Christensen BC, Marsit CJ, Nelson $\mathrm{HH}$, et al. DNA methylation arrays as surrogate measures of cell mixture distribution. BMC Bioinform. 2012;13:86.

37. Desikan RS, Ségonne F, Fischl B, Quinn BT, Dickerson BC, Blacker D, et al. An automated labeling system for subdividing the human cerebral cortex on MRI scans into gyral based regions of interest. Neuroimage. 2006;31:968-80.

38. Faul F, Erdfelder E, Buchner A, Lang AG. Statistical power analyses using G*Power 3.1: tests for correlation and regression analyses. Behav Res Methods. 2009;41:1149-60.

39. Benjamini $Y$, Hochberg $Y$. Controlling the false discovery rate: a practical and powerful approach to multiple testing. J R Stat Soc Ser B. 1995;57:289-300.

40. Uddin M, Aiello AE, Wildman DE, Koenen KC, Pawelec G, de Los Santos R, et al. Epigenetic and immune function profiles associated with posttraumatic stress disorder. Proc Natl Acad Sci USA. 2010;107:9470-5.

41. Goldmann E, Aiello A, Uddin M, Delva J, Koenen K, Gant LM, et al. Pervasive exposure to violence and posttraumatic stress disorder in a predominantly African American Urban Community: the Detroit Neighborhood Health Study. J Trauma Stress. 2011;24:747-51.

42. Breslau N, Kessler RC, Chilcoat HD, Schultz LR, Davis GC, Andreski P. Trauma and posttraumatic stress disorder in the community: the 1996 Detroit Area Survey of Trauma. Arch Gen Psychiatry. 1998;55:626-32.

43. Roberts AL, Koenen KC, Chen Q, Gilsanz P, Mason SM, Prescott J, et al. Posttraumatic stress disorder and accelerated aging: PTSD and leukocyte telomere length in a sample of civilian women. Depress Anxiety. 2017;34:391-400.

44. King KS, Kozlitina J, Rosenberg RN, Peshock RM, McColl RW, Garcia CK. Effect of leukocyte telomere length on total and regional brain volumes in a large population-based cohort. JAMA Neurol. 2014;71:1247-54.

45. Wolf EJ, Sadeh N, Leritz EC, Logue MW, Stoop TB, McGlinchey R, et al. Posttraumatic stress disorder as a catalyst for the association between metabolic syndrome and reduced cortical thickness. Biol Psychiatry. 2016;80:363-71.

46. Fjell AM, Grydeland H, Krogsrud SK, Amlien I, Rohani DA, Ferschmann L, et al. Development and aging of cortical thickness correspond to genetic organization patterns. Proc Natl Acad Sci USA. 2015;112:15462-7.

47. Storsve AB, Fjell AM, Tamnes CK, Westlye LT, Overbye K, Aasland HW, et al. Differential longitudinal changes in cortical thickness, surface area and volume across the adult life span: regions of accelerating and decelerating change. J Neurosci. 2014;34:8488-98.

48. Rolls ET. The orbitofrontal cortex and reward. Cereb cortex. 2000;10:284-94.

49. Bremner JD, Vythilingam M, Vermetten E, Nazeer A, Adil J, Khan S, et al. Reduced volume of orbitofrontal cortex in major depression. Biol Psychiatry. 2002;51:273-79.
50. Lai T-J, Payne ME, Byrum CE, Steffens DC, Krishnan KRR. Reduction of orbital frontal cortex volume in geriatric depression. Biol Psychiatry. 2000;48:971-75.

51. Leech R, Braga R, Sharp DJ. Echoes of the brain within the posterior cingulate cortex. J Neurosci. 2012;32:215-22.

52. Chang $Y-L$, Jacobson MW, Fennema-Notestine $C$, Hagler Jr DJ, Jennings RG, Dale $A M$, et al. Level of executive function influences verbal memory in amnestic mild cognitive impairment and predicts prefrontal and posterior cingulate thickness. Cereb Cortex. 2009;20:1305-13.

53. Lehmann M, Rohrer JD, Clarkson MJ, Ridgway GR, Scahill RI, Modat M, et al. Reduced cortical thickness in the posterior cingulate gyrus is characteristic of both typical and atypical Alzheimer's disease. J Alzheimer's Dis. 2010;20:587-98.

54. Soehnlein O, Steffens S, Hidalgo A, Weber C. Neutrophils as protagonists and targets in chronic inflammation. Nat Rev Immunol. 2017;17:248-61.

55. Prame Kumar K, Nicholls AJ, Wong CHY. Partners in crime: neutrophils and monocytes/macrophages in inflammation and disease. Cell Tissue Res. 2018;371:551-65.

56. Wang $T$, Jiang $C Q$, Xu L, Zhang WS, Zhu F, Jin YL, et al. White blood cell count and all-cause and cause-specific mortality in the Guangzhou biobank cohort study. BMC Public Health. 2018;18:1232.

57. Leng SX, Xue QL, Huang Y, Ferrucci L, Fried LP, Walston JD. Baseline total and specific differential white blood cell counts and 5-year all-cause mortality in community-dwelling older women. Exp Gerontol. 2005;40:982-7.

58. Kim KI, Lee J, Heo NJ, Kim S, Chin HJ, Na KY, et al. Differential white blood cell count and all-cause mortality in the Korean elderly. Exp Gerontol. 2013;48: 103-8.

59. Fest J, Ruiter TR, Groot Koerkamp B, Rizopoulos D, lkram MA, van Eijck CHJ, et al. The neutrophil-to-lymphocyte ratio is associated with mortality in the general population: The Rotterdam Study. Eur J Epidemiol. 2019;34:463-70.

60. Miller MW, Lin AP, Wolf EJ, Miller DR. Oxidative stress, inflammation, and neuroprogression in chronic PTSD. Harv Rev Psychiatry. 2018;26:57-69.

61. Horvath S, Gurven M, Levine ME, Trumble BC, Kaplan H, Allayee H, et al. An epigenetic clock analysis of race/ethnicity, sex, and coronary heart disease. Genome Biol. 2016;17:171.

62. Schnurr PP, Spiro A 3rd, Paris AH. Physician-diagnosed medical disorders in relation to PTSD symptoms in older male military veterans. Health Psychol. 2000;19:91-7.

63. Schlenger WE, Corry NH, Williams CS, Kulka RA, Mulvaney-Day N, DeBakey S, et al. A prospective study of mortality and trauma-related risk factors among a nationally representative sample of Vietnam veterans. Am J Epidemiol. 2015;182:980-90. 